\title{
ORIGINAL
}

\section{EXCESO DE MORTALIDAD PRECOZ RELACIONADO CON LA GRIPE EN ESPAÑA DURANTE UN PERIODO INTERPANDÉMICO $(*)$}

\section{Lorena Simón Méndez (1), Teresa López-Cuadrado (1), Noemí López Perea (2), Amparo Larrauri Cámara (1,3) y Salvador de Mateo Ontañón $(1,3)$}

(1) Centro Nacional de Epidemiología, Instituto de Salud Carlos III, Madrid, España.

(2) Programa de Epidemiología Aplicada al Campo (PEAC).

(3) Ciber de Epidemiología y Salud Pública, CIBERESP, España.

(*) Programa de Investigación de la Gripe A (H1N1), Instituto de Salud Carlos III (GR09/0018)

Conflicto de interés: Ninguno.

\section{RESUMEN}

Fundamentos: El indicador Años Potenciales de Vida Perdidos (APVP) ha sido utilizado con frecuencia en análisis de mortalidad pre$\mathrm{coz}, \mathrm{y}$ recientemente ha servido para estimar el impacto de la última pandemia de gripe A(H1N1)pdm09. El objetivo de este estudio ha sido estimar los excesos de defunciones por gripe y neumonía (GyN) en España y los APVP durante el periodo 1980-2008, valorando la mortalidad atribuible a gripe en función del tipo/subtipo de virus predominante.

Métodos: Los excesos de defunciones mensuales se calcularon con modelos de regresión cíclica. El cálculo de APVP se realizó como el producto del número de excesos de defunciones por la diferencia entre la esperanza de vida al nacer y los años vividos para cada grupo de edad. El análisis de la variación entre los excesos de defunciones por GyN y APVP, según el tipo/subtipo de virus de la gripe predominante, se llevó a cabo con un análisis de regresión de Poisson.

Resultados: En las temporadas con predominio del virus de la gripe $\mathrm{A}(\mathrm{H} 3)$ el promedio de excesos de defunciones por GyN se estimó en 1.348 y de APVP en 5.297, mientras que en las temporadas con predominio de $\mathrm{A}(\mathrm{H} 1)$ o $\mathrm{B}$ el promedio de excesos por las mismas causas fue de 648 y de APVP de 2.885. Las razones de tasas ajustadas de excesos $(2,11 ;$ IC- $95 \%=2,05-2,16)$ y de APVP $(1,86$; IC- $95 \%=1,83-1,88)$ señalaron que las frecuencias relativas de ambos indicadores son significativamente mayores en las temporadas con predominio de virus de la gripe $\mathrm{A}(\mathrm{H} 3)$.

Conclusiones: Los excesos de defunciones y APVP se duplicaron al comparar las temporadas con predominio del subtipo A(H3) frente al resto de virus de la gripe.

Palabras clave: Años Potenciales de Vida Perdidos. Exceso de mortalidad. Mortalidad Precoz. Gripe. España.

Correspondencia

Lorena Simón Méndez

Centro Nacional de Epidemiología

Instituto de Salud Carlos III

Av/. Monforte de Lemos, 5

28029 Madrid

lorenas@isciii.es

\section{ABSTRACT \\ Premature Mortality Excess Related to Influenza in Spain during an Interpandemic Period}

Background: The indicator of Potential Years of Life Lost (PYLL) has been frequently used to analysis of premature mortality and recently has been used to estimate the impact of the last influenza A(H1N1)pdm09 pandemic. The aim of this study was to estimate the excess deaths from pneumonia and influenza (P\&I) in Spain and the PYLL during the period 1980-2008, measuring the mortality attributable to influenza regarding the type/subtype of influenza dominant in each season.

Methods: Monthly excess deaths were calculated with cyclical regression models. The PYLL calculation was performed as the product of the number of excess deaths and the difference between life expectancy at birth and years lived for each age group. The analysis of the variation between P\&I excess deaths and PYLL, depending on the predominant influenza virus type/subtype was carried out with a Poisson regression analysis.

Results: In seasons dominated by influenza virus A(H3) the average P\&I excess deaths was estimated at 1,348, and for PYLL in 5.297, while in seasons dominated by $\mathrm{A}(\mathrm{H} 1)$ or $\mathrm{B}$ the average P\&I excess deaths was 648 , and for PYLL 2.885. The adjusted rate ratios of excess $(2.11$, CI-95\% $=2.05-2.16)$ and PYLL $(1.86$, CI-95\%=1.83-1.88) indicate that the relative frequencies for both indicators are significantly larger in seasons dominated by influenza virus $\mathrm{A}(\mathrm{H} 3)$.

Conclusions: Excess deaths and PYLL doubled when comparing seasons predominantly subtype $\mathrm{A}(\mathrm{H} 3)$ and other influenza viruses

Key words: Potential Years of Life Lost. Excess mortality. Premature mortality. Influenza. Spain. 


\section{INTRODUCCIÓN}

La concepción original del indicador Años Potenciales de Vida Perdidos (APVP) tuvo mucho que ver con el intento de comparar la importancia relativa de las diferentes causas de muerte de una población en particular ${ }^{1,2}$, primando aquellas que presentaban una mayor frecuencia en las primeras etapas de la vida. A la hora de priorizar problemas de salud con este nuevo indicador no solo se tenía en cuenta la magnitud de la mortalidad de los distintos problemas, reflejada por el número de defunciones, sino que se añadía el concepto de trascendencia, en el sentido de considerar de mayor importancia la defunción de una persona de menor edad. Por esta razón, dicho indicador ha sido utilizado con frecuencia en los análisis de mortalidad pre$\mathrm{coz}^{3,4}$ y recientemente ha servido para estimar el impacto de la última pandemia de gripe $\mathrm{A}(\mathrm{H} 1 \mathrm{~N} 1) \mathrm{pdm} 09$, caracterizada en muchos países por una mayor mortalidad atribuible en población joven, produciendo un incremento no tanto de las defunciones sino de los APVP ${ }^{5-8}$. Este desplazamiento de la mortalidad atribuible a gripe a edades más jóvenes durante los periodos pandémicos de gripe es algo conocido y ha sido observado con regularidad en el estudio de las pandemias acontecidas en el siglo pasa$\mathrm{do}^{9}$, pero no se ha descrito en los periodos interpandémicos ni durante las ondas epidémicas estacionales, en los que la mayoría de las defunciones ocurren en personas mayores de 65 años ${ }^{10,11}$.

Ahora bien, uno de los principales problemas a la hora de evaluar la mortalidad por gripe en una población es que el número de defunciones atribuibles a la gripe es muy difícil de estimar directamente, dado que las infecciones por el virus de la gripe no son confirmadas habitualmente en laboratorio y el diagnóstico de gripe aparece con rara frecuencia en el apartado de causa fundamental de los certificados de defun- ción. Por otra parte, muchas muertes relacionadas con la infección gripal ocurren por complicaciones secundarias cuando ya es muy difícil detectar signos de infección por los virus de la gripe y ha sido olvidada la causa que motivó la descompensación de la enfermedad de base del paciente ${ }^{11,12}$. Por estas razones la mortalidad atribuible a la gripe habitualmente es determinada indirectamente, con el uso de modelos que estiman el exceso de defunciones observadas en los períodos invernales por encima de un nivel basal de mortalidad esperada en ausencia de circulación de virus de la gri$\mathrm{pe}^{13}$, considerándose que las defunciones por GyN son las que de forma más específica se asocian con la gripe ${ }^{14}$.

El objetivo principal de este estudio fue estimar los excesos de defunciones por gripe y neumonía en España y los APVP de esos excesos durante un periodo interpandémico (1980-2008) y valorar con ambos indicadores la variación de la mortalidad atribuible a gripe en función del tipo/subtipo predominante de virus de la gripe en circulación.

\section{MATERIAL Y MÉTODOS}

La información sobre defunciones en España durante el periodo estudiado (19802008) se obtuvo del Instituto Nacional de Estadística (INE), incluyendo como muertes por gripe y neumonía (GYN) las codificadas como 480-488 en la novena revisión de la Clasificación Internacional de Enfermedades y Causas de Muerte (CIE 9 $\left.{ }^{\mathrm{a}}\right)$, vigente durante los años 1980-1998, y las codificadas como J09-J18 en la décima revisión de dicha Clasificación (CIE 10a), utilizada desde el año 1999 a la actualidad. El análisis de la mortalidad se efectuó no en años naturales sino en temporadas epidemiológicas (periodo comprendido entre el mes de octubre de un año y el mes de septiembre del año siguiente), de acuerdo con el patrón de distribución estacional de las defunciones por las causas estudiadas, y 
tomando como población de referencia las proyecciones calculadas por el INE, corregidas para el último periodo $(2007 / 08)$ por el Censo de Población de 2001.

Para estimar el exceso de defunciones globales y por GyN, se cuantificó en primer lugar la mortalidad esperada en ausencia de actividad gripal, aplicando un modelo de regresión cíclica ${ }^{13,15}$ (modelo de Serfling adaptado) a los datos ajustados de mortalidad mensual registrada por ese grupo de causas, una vez eliminada la tendencia y excluyendo los valores de los meses de diciembre a abril de cada temporada. Posteriormente se calcularon las tasas mensuales de exceso de mortalidad como diferencia entre tasas observadas y esperadas durante los meses epidémicos y el exceso de mortalidad por temporada como suma de los excesos mensuales. El modelo fue aplicado sucesivamente a cada uno de los nueve grupos de edad considerados ( $<1$ año; 1-4; 5$14 ; 15-24 ; 25-34 ; 35-44 ; 45-54 ; 55-64$ y $>64$ años), calculándose los excesos globales para cada temporada y las tasas de exceso por 100.000 habitantes que se presentan estandarizadas por edad con el uso del método directo de ajuste.

Con arreglo a la esperanza de vida al nacimiento de la población española calculada por el INE, el cálculo de APVP se realizó para cada grupo de edad como el producto del número de excesos de defunciones obtenido en el análisis anterior por la diferencia entre la esperanza de vida al nacer y los años vividos por el grupo de edad correspondiente (punto medio del intervalo de edad considerado, salvo para el último grupo de edad que se fijó en 75 años). Para el ajuste por edad de las tasas de APVP se aplicó el método de estandarización directa propuesto por Romeder y McWhinnie ${ }^{1}$.

La información sobre el tipo de virus de gripe dominante en cada temporada se obtuvo del Sistema de Vigilancia de la Gri- pe en España ${ }^{16}$, en el periodo 1996-2008, y del Sistema de Información Microbiológi$\mathrm{ca}^{17}$ para las temporadas previas. En las temporadas en las que se observó una circulación mixta de virus de la gripe, el predominio se atribuyó al virus cuya frecuencia relativa fue mayor en los aislamientos notificados por los laboratorios.

El análisis de la variación existente entre los excesos de defunciones por GyN y APVP según el tipo/subtipo de virus de la gripe predominante, se llevó a cabo con un análisis de regresión de Poisson, mientras que la relación entre ambos indicadores se estableció con el uso del coeficiente de correlación de rangos de Spearman (rs).

\section{RESULTADOS}

En las 28 temporadas interpandémicas estudiadas (1980/81-2007/08) se registró en España un total de 239.581 defunciones por GyN, lo que supone que este conjunto de causas signifique en ese periodo el 2,4\% del total de defunciones y el $24,1 \%$ de las defunciones por enfermedades respiratorias, si bien esos porcentajes varíaron de forma significativa en las distintas temporadas. Así, en relación con el total de defunciones, la frecuencia relativa de muertes por GyN osciló en las temporadas analizadas en un rango de $2,0 \%$ y $3,9 \%$, mientras que en relación con las enfermedades respiratorias el rango fue entre $19,0 \%$ y $40,2 \%$.

La mortalidad por GyN tuvo un comportamiento estacional muy definido con valores máximos en los meses invernales, y su distribución por grupos de edad fue también muy regular afectando principalmente a población mayor de 64 años. En este grupo de edad fue donde se dio la gran mayoría de las muertes por GyN, representando el 89,4\% de las defunciones (rango en las diferentes temporadas: $82,6 \%-92,6 \%$ ).

Para el total de la población, el modelo de regresión empleado estimó el exceso de mor- 
talidad por GyN que se representa en la figura 1 , con picos cíclicos de tasas de excesos de defunciones por GyN. Cuando se emplearon modelos para cada uno de los grupos de edad considerados se obtuvieron las cifras mostradas en la tabla 1. El rango de excesos de defunciones por GyN fue de 0 defunciones para la temporada 2000/01 a 4.389 en la temporada 1980/81, mientras que para los APVP estuvo comprendido entre 0 para la temporada $2000 / 01$ y 14.204 para $2004 / 05$. Junto al número de excesos de defunciones y APVP para cada una de las temporadas analizadas, se presentan las tasas ajustadas por 100.000 habitantes, que alcanzaron sus valores máximos en la temporada 1980/81, con valores de 11,63 y 36,33 respectivamente. Estas tasas presentaron un patrón de evolución temporal muy similar (figura 2), con un alto grado de correlación ( $\mathrm{rs}=0,988 ; \mathrm{P}<0,00001)$ y picos más pronunciados para las temporadas $1980 / 81,1982 / 83,1998 / 99$ y 2004/05.
Llama la atención que en la mayoría de las temporadas con elevadas tasas de excesos de defunciones y APVP (tabla 1), el virus de la gripe que circuló predominantemente fue el subtipo $\mathrm{A}(\mathrm{H} 3)$. Tanto es así que el promedio de excesos de defunciones por GyN en las 16 temporadas con predominio de $\mathrm{A}(\mathrm{H} 3)$ se estimó en 1.348, frente a 648 en las 12 temporadas con predominio de $\mathrm{A}(\mathrm{H} 1)$ o B. Pero un aumento de similar magnitud se observó también con los APVP: en la tabla 2 se presentan razones de tasas ajustadas de excesos y APVP y vemos que tanto la razón de excesos $(2,11$; IC-95\% $=2,05$ $2,16)$ como la de $\operatorname{APVP}(1,86$; IC$95 \%=1,83-1,88)$ señalaron de forma paralela que las frecuencias relativas de ambos indicadores fueron significativamente mayores en las temporadas con predominio de virus de la gripe $\mathrm{A}(\mathrm{H} 3)$.

Figura 1

Tasas mensuales de mortalidad por gripe y neumonía en España. Enero de 1980-Diciembre de 2008. Tasas basales determinadas por el modelo Serfling

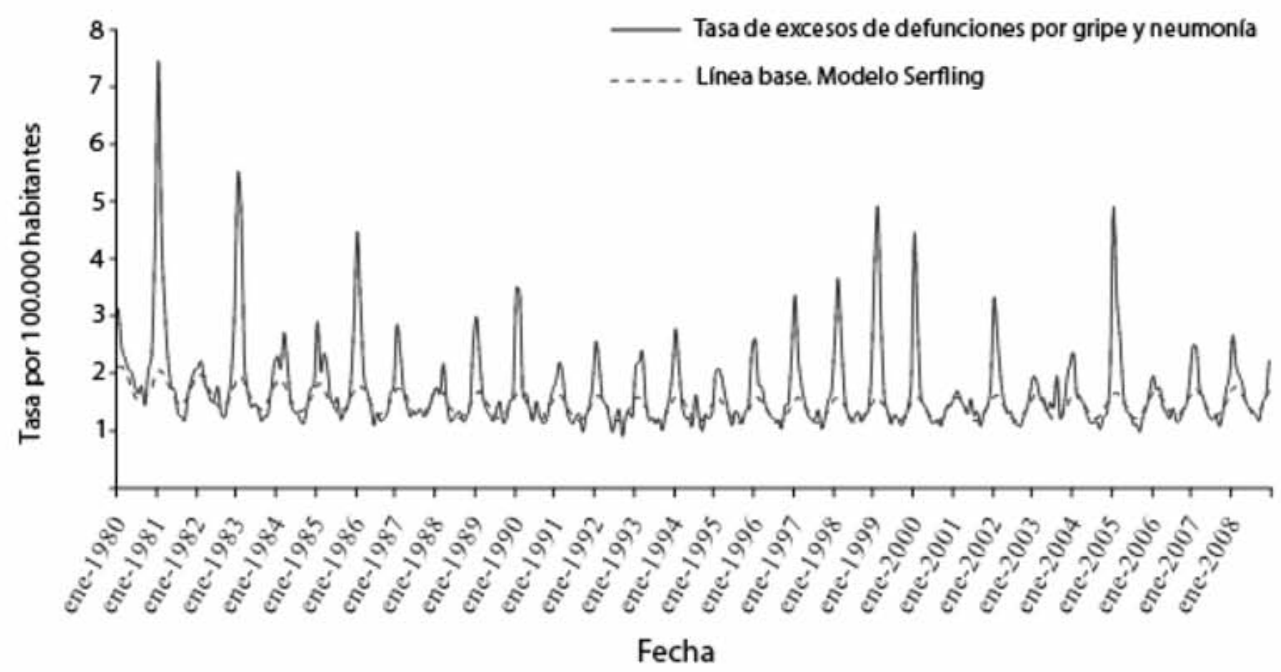


Tabla 1

Tasa ajustada de excesos de defunciones y de APVP por gripe y neumonía /100.000 habitantes. España. Temporadas 1980/81 - 2007/08

\begin{tabular}{|c|c|c|c|c|c|}
\hline Temporadas & Tipo/subtipo & $\begin{array}{c}\text { Excesos de } \\
\text { defunciones por } \\
\text { G\&N }\end{array}$ & $\begin{array}{l}\text { Tasa ajustada de exce- } \\
\text { sos de defunciones por } \\
\text { G\&N/100.000 hab* }\end{array}$ & $\begin{array}{l}\text { APVP } \\
\text { por } \\
\text { G\&N }\end{array}$ & $\begin{array}{c}\text { Tasa ajustada de } \\
\text { APVP } \\
\text { por } \\
\text { G\&N/100.000 hab* }\end{array}$ \\
\hline $1980 / 81$ & $\mathrm{~A}(\mathrm{H} 3 \mathrm{~N} 2)$ & 4.389 & 11,63 & 13.713 & 36,33 \\
\hline $1981 / 82$ & $\mathrm{~A}(\mathrm{H} 1 \mathrm{~N} 1)$ & 141 & 0,37 & 1.436 & 3,85 \\
\hline $1982 / 83$ & $\mathrm{~A}(\mathrm{H} 3 \mathrm{~N} 2) / \mathrm{B}$ & 3.076 & 7,86 & 10.241 & 27 \\
\hline $1983 / 84$ & $\mathrm{~A}(\mathrm{H} 1 \mathrm{~N} 1)$ & 870 & 2,2 & 3.074 & 8,1 \\
\hline $1984 / 85$ & $\mathrm{~A}(\mathrm{H} 3 \mathrm{~N} 2)$ & 920 & 2,26 & 3.045 & 7,95 \\
\hline $1985 / 86$ & $\mathrm{~A}(\mathrm{H} 3 \mathrm{~N} 2) / \mathrm{B}$ & 2.138 & 5,11 & 6.290 & 15,57 \\
\hline $1986 / 87$ & $\mathrm{~A}(\mathrm{H} 1 \mathrm{~N} 1)$ & 673 & 1,58 & 3.172 & 8,36 \\
\hline $1987 / 88$ & $\mathrm{~B}$ & 210 & 0,49 & 762 & 2,63 \\
\hline $1988 / 89$ & $\mathrm{~A}(\mathrm{H} 1 \mathrm{~N} 1)$ & 1.082 & 2,4 & 4.047 & 9,66 \\
\hline $1989 / 90$ & $\mathrm{~A}(\mathrm{H} 3 \mathrm{~N} 2) / \mathrm{B}$ & 1.372 & 2,97 & 5.429 & 13,33 \\
\hline $1990 / 91$ & $\mathrm{~B}$ & 426 & 0,9 & 1.647 & 4,18 \\
\hline $1991 / 92$ & $\mathrm{~A}(\mathrm{H} 3 \mathrm{~N} 2)$ & 491 & 1,01 & 2.287 & 5,06 \\
\hline $1992 / 93$ & $\mathrm{~A}(\mathrm{H} 3 \mathrm{~N} 2)$ & 722 & 1,46 & 3.280 & 7,35 \\
\hline $1993 / 94$ & $\mathrm{~A}(\mathrm{H} 3 \mathrm{~N} 2)$ & 666 & 1,31 & 2.664 & 5,53 \\
\hline $1994 / 95$ & $\mathrm{~A}(\mathrm{H} 3 \mathrm{~N} 2) / \mathrm{B}$ & 464 & 0,89 & 1.715 & 3,4 \\
\hline $1995 / 96$ & $\mathrm{~A}(\mathrm{H} 3 \mathrm{~N} 2)$ & 759 & 1,43 & 2.869 & 5,62 \\
\hline $1996 / 97$ & $\mathrm{~A}(\mathrm{H} 3 \mathrm{~N} 2) / \mathrm{B}$ & 1.268 & 2,34 & 5.133 & 10,14 \\
\hline $1997 / 98$ & $\mathrm{~A}(\mathrm{H} 3 \mathrm{~N} 2)$ & 1.547 & 2,78 & 6.711 & 12,57 \\
\hline $1998 / 99$ & $\mathrm{~B}$ & 2.630 & 4,62 & 11.765 & 21,53 \\
\hline $1999 / 00$ & $\mathrm{~A}(\mathrm{H} 1 \mathrm{Nl})$ & 1.726 & 2,97 & 8.105 & 14,62 \\
\hline $2000 / 01$ & $\mathrm{~A}(\mathrm{H} 1 \mathrm{Nl})$ & 0 & - & 0 & - \\
\hline $2001 / 02$ & $\mathrm{~A}(\mathrm{H} 3 \mathrm{~N} 2)$ & 1.038 & 1,71 & 5.504 & 9,61 \\
\hline $2002 / 03$ & $\mathrm{~B}$ & 5 & 0,01 & 244 & 0,45 \\
\hline $2003 / 04$ & $\mathrm{~A}(\mathrm{H} 3 \mathrm{~N} 2)$ & 249 & 0,4 & 1.340 & 2,24 \\
\hline $2004 / 05$ & $\mathrm{~A}(\mathrm{H} 3 \mathrm{~N} 2)$ & 2.455 & 3,86 & 14.204 & 22,84 \\
\hline $2005 / 06$ & $\mathrm{~A}(\mathrm{H} 1 \mathrm{~N} 1) / \mathrm{B}$ & 1 & 0 & 39 & 0,14 \\
\hline $2006 / 07$ & $\mathrm{~A}(\mathrm{H} 3 \mathrm{~N} 2)$ & 16 & 0,03 & 328 & 0,67 \\
\hline $2007 / 08$ & $\mathrm{~B} / \mathrm{A}(\mathrm{H} 1 \mathrm{~N} 1)$ & 11 & 0,02 & 346 & 0,69 \\
\hline
\end{tabular}

* Ajustada a la población del año 1981 
Figura 2

Tasa de excesos de defunciones y APVP por gripe y neumonía, ajustada por edad.

Temporadas 1980/81 - 2007/08

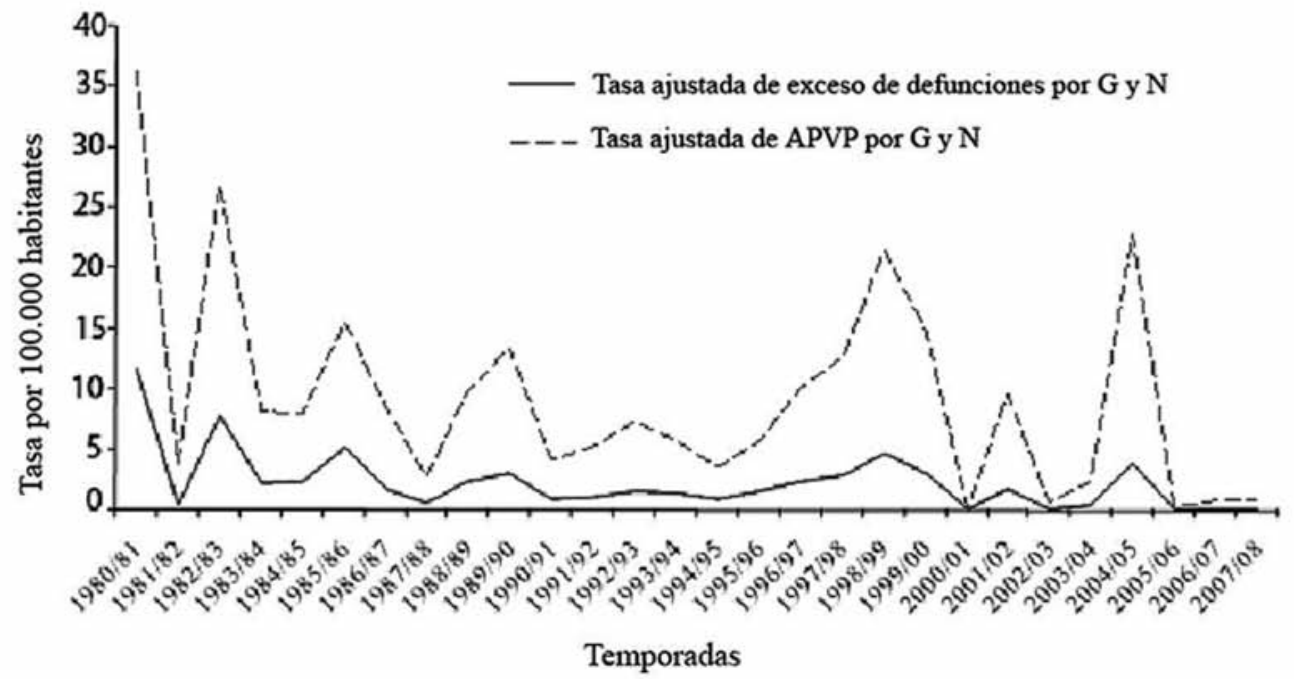

Tabla 2

Razón de tasas de excesos de defunciones y de APVP por gripe y neumonía.

Temporadas 1980/81 - 2007/08

\begin{tabular}{|l|c|c|}
\hline Tipo/subtipo dominante & $\mathbf{A}(\mathbf{H} 1 \mathbf{N} 1) / \mathbf{B}$ & $\mathbf{A}(\mathbf{H} 3 \mathbf{N} 2)$ \\
\hline Excesos & $\mathbf{7 . 7 7 5}$ & $\mathbf{2 1 . 5 6 9}$ \\
\hline Promedio por temporada & 648 & 1.348 \\
\hline Tasas de excesos $/ 100.000 \mathrm{~h}$. & 1,61 & 3,37 \\
\hline Razón de tasas de excesos* & 1 & $2,11(2,05-2,16)$ \\
\hline APVP & $\mathbf{3 4 . 6 2 4}$ & $\mathbf{8 4 . 7 4 3}$ \\
\hline Promedio por temporada & 2.885 & 5.297 \\
\hline Tasas de APVP / $100.000 \mathrm{~h}$. & 7,16 & 13,24 \\
\hline Razón de tasas de APVP* & 1 & $1,86(1,83-1,88)$ \\
\hline
\end{tabular}

* Ajustada por grupos de edad 


\section{DISCUSIÓN}

El trabajo presenta estimaciones del exceso de defunciones por GyN y los APVP causados por ese exceso, atribuibles a la gripe en España, en un largo periodo de tiempo caracterizado por la ausencia de pandemias de gripe, y analiza la variabilidad de ambos indicadores según la circulación predominante de los distintos tipos/subtipos de virus de la gripe.

El uso de modelos estadísticos para estimar la mortalidad atribuible a la gripe constituye una importante herramienta desde su introducción en la metodología epidemiológica hace más de un siglo $^{18}$. El concepto de exceso de defunciones estimado por estos modelos se justifica empíricamente por las numerosas observaciones de incremento de muertes durante las ondas epidémicas de gripe que suelen atribuirse a neumonía u otra serie de complicaciones secundarias $^{19}$, por lo que al analizar únicamente el exceso de defunciones por GyN, como hemos hecho en este trabajo, es muy posible que no se esté midiendo de forma sensible la carga total de la gripe en la mortalidad de la población española, pero sí evaluando de forma específica la gravedad de las epidemias de gripe ${ }^{14}$.

Variaciones en el número de defunciones relacionadas con la gripe no sólo pueden darse por el conjunto de causas de mortalidad analizadas sino también por el tipo de modelo utilizado. Una crítica frecuente al empleo del modelo de regresión usado en nuestro análisis para la predicción de los niveles basales de mortalidad y la subsiguiente estimación de excesos de defunciones es que los denominados periodos epidémicos no son validados por ningún tipo de evidencia virológica de actividad de virus gri$\mathrm{pal}^{14,20}$. De hecho, podríamos decir que lo que constituye la mayor ventaja de este modelo, pues es aplicable a situacio- nes en las que no se dispone de información virológica, se erige a su vez en uno de sus puntos débiles. Por otra parte, también son frecuentes, en comparación con el uso alternativo de modelos lineales generalizados (GLM), que admiten la inclusión de covariables que miden la actividad del virus de la gripe u otros virus respiratorios ${ }^{21}$, críticas centradas en la sobreestimación de excesos obtenidos con el modelo utilizado en este trabajo $^{22}$. Sin embargo, a pesar de sus limitaciones, este tipo de modelo de regresión cíclica, derivado de la metodología propuesta por Serfling hace casi 40 años, sigue siendo uno de los métodos más usado para la estimación de excesos de defunciones atribuibles a la gripe $e^{5,15,23}$.

Salvo en periodos de pandemia gripal, cuando es consistente el hecho de un desplazamiento de la mortalidad asociada a gripe a edades más jóvenes ${ }^{13,14}$ y el consiguiente aumento de los APVP, los excesos de defunciones son muy regulares en cuanto a su distribución por edad, por lo que dado el carácter interpandémico del periodo estudiado no es de extrañar la estrecha correlación existente entre ambos indicadores que hemos ha1lado. Ello supone que en los periodos interpandémicos puede emplearse indistintamente uno u otro indicador para evaluar la variación existente en la mortalidad relacionada con la gripe según el tipo/subtipo de virus de la gripe predominante. En el periodo analizado, las temporadas con estimaciones altas de excesos de defunciones o APVP tuvieron predominio de subtipo $\mathrm{A}(\mathrm{H} 3)$, llegando a duplicarse tanto los excesos de defunciones como los APVP cuando se comparaban temporadas con predominio del subtipo $\mathrm{A}(\mathrm{H} 3)$ con temporadas de predominio de $\mathrm{A}(\mathrm{H} 1)$ o $\mathrm{B}$. Esta variación de la mortalidad dependiendo del tipo/subtipo de virus de la gripe circulante, con mayor mortalidad en las temporadas en que el subtipo $\mathrm{A}(\mathrm{H} 3)$ es predominante, ya que se 
ha observado regularmente en los diferentes estudios que han analizado este hecho en distintas partes del mundo ${ }^{10,14,24,25}$.

En conclusión, el modelo utilizado ofrece una estimación del impacto de las ondas estacionales de gripe en el periodo interpandémico 1980-2008. Los excesos de defunciones por gripe y neumonía en España y los APVP en dicho periodo interpandémico se duplicaron al comparar las temporadas con predominio del subtipo $\mathrm{A}(\mathrm{H} 3)$ frente al resto de virus de la gripe.

\section{AGRADECIMIENTOS}

A todos los integrantes del Sistema de Vigilancia de gripe en España. Así mismo, los autores agradecen la contribución de Caterina Rizzo en el diseño del modelo de Serfling utilizado en este artículo.

\section{BIBLIOGRAFÍA}

1. Romeder J-M, McWhinnie JR. Potential years of life lost between ages 1 and 70: an indicator of premature mortality for health planning. Int J Epidemiol. 1977;6 (2):143-51.

2. Dempsey M. Decline in tuberculosis; the death rate fails to tell the entire story. Am Rev Tuberc. 1947;56(2):157-64.

3. Premature mortality in the United States: public health issues in the use of years of potential life lost. MMWR Morb Mortal Wkly Rep. 1986;35(2 Suppl):1S-11S.

4. Introduction to Table V. Premature deaths, monthly mortality, and monthly physician contacts--United States. MMWR Morb Mortal Wkly Rep. 1982;31(9):109-10.

5.Viboud C, Miller M, Olson D, Osterholm M, Simonsen L. Preliminary Estimates of Mortality and Years of Life Lost Associated with the 2009 A/H1N1 Pandemic in the US and Comparison with Past Influenza Seasons. PLoS Curr. 2010;RRN1153.

6. Butler D. Portrait of a year-old pandemic. Nature. 2010; 464:1112-3

7. Simón L, de Mateo S, Larrauri A, Jiménez-Jorge S, Vaque J, Perez S. Transmisibilidad y gravedad de la pandemia de gripe A(H1N1)2009 en España. Gac Sanit. 2011;25 (4):296-302.
8. Charu V, Chowell G, Palacio Mejia LS, EchevarríaZuno S, Borja-Aburto VH, Simonsen L, Miller MA, Viboud C. Mortality burden of the A/H1N1 pandemic in Mexico: a comparison of deaths and years of life lost to seasonal influenza. Clin Infect Dis. 2011;53 (10):985-93.

9. Simonsen L, Clarke MJ, Schonberger LB, Arden NH, Cox NJ, Fukuda K. Pandemic versus epidemic influenza mortality: a pattern of changing age distribution. J Infect. 1998;178:53-60.

10. Cohen C, Simonsen L, Kang JW, Miller M, McAnerney J, Blumberg L, et al. Elevated influenza-related excess mortality in South African elderly individuals, 1998-2005. Clin Infect Dis. 2010;51(12):1362-9.

11. Pitman RJ, Melegaro A, Gelb D, Siddiqui MR, Gay NJ, Edmunds WJ. Assessing the burden of influenza and other respiratory infections in England and Wales. J Infect. 2007;54 (6):530-8.

12. WHO. Comparing deaths from pandemic and seasonal influenza Pandemic (H1N1) 2009. Pandemic (H1N1) 2009 briefing note 20 2009. Disponible en:

http://www.who.int/csr/disease/swineflu/notes/briefing_20091222/en/index.html

13. Rizzo C, Bella A, Viboud C, Simonsen L, Miller MA, Rota MC, et al. Trends for influenza-related deaths during pandemic and epidemic seasons, Italy, 1969-2001. Emerg Infect Dis. 2007;13 (5):694-9.

14. Simonsen L, Clarke MJ, Williamson GD, Stroup DF, Arden NH, Schonberger LB. The impact of influenza epidemics on mortality: introducing a severity index. Am J Public Health. 1997;87 (12):1944-50.

15. Serfling RE. Methods for current statistical analysis of excess pneumonia-influenza deaths. Public Health Rep. 1963;78 (6):494-506.

16.Centro Nacional de Epidemiología. Instituto de Salud Carlos III. Informes de vigilancia de gripe en España. Sistema de Vigilancia de la Gripe en España (SVGE). Disponible en: http://vgripe.isciii.es/gripe, http://cne-gripe-infsemanal. isciii.es.

17. Centro Nacional de Epidemiología. Instituto de Salud Carlos III. Sistema de Información Microbiológica (SIM) Disponible en: http://www.isciii.es/ISCIII/es/contenidos/fd-servicios-cientifico-tecnicos/fd-vigilancias-alertas/sistema-informacion-microbiologica.shtml.

18. Humphrey N, ed. Vital Statistics: Memorial Volume of Selections from the Writings of William Farr. Part 4. London: Sanitary Institute of Great Britain; $1885: 330$ 
19. Housworth J, Langmuir AD. Excess mortality from epidemic influenza, 1957-1966. Am J Epidemiol. 1974; 100:40-8

20. Glezen P, Ardath AP, Snyder DN, Downs TD. Mortality and influenza. J Infect Dis. 1982;146:313-21.

21. León Gómez I, Flores Segovia VM, Jiménez Jorge S, Larrauri Cámara A, Palmera Suárez R, Simón Soria F. Excesos de mortalidad en España durante la transmisión de gripe pandémica en el año 2009. Rev Esp Salud Pública. 2010;84(5):589-96.

22. López-Cuadrado T, de Mateo S, Jiménez-Jorge S, Savulescu C, Larrauri A. Influenza-related mortality in S p a in, $1999-2005$. G a c S anit. 2012 . doi:10.1016/j.gaceta.2011.09.033.

23. Rizzo C, Viboud C, Montomoli E, Simonsen L, Miller MA. Influenza-related mortality in the Italian elderly: no decline associated with increasing vaccination coverage. Vaccine. 2006;24(42-43):6468-75.

24. CDC. Estimates of deaths associated with seasonal influenza - United States, 1976-2007. MMWR Morb Mortal Wkly Rep. 2010;59(33):1057-62.

25. Nunes B, Viboud C, Machado A, Ringholz C, Rebelo-de-Andrade H, Nogueira P, et al. Excess mortality associated with influenza epidemics in Portugal, 1980 to 2004. PLoS ONE. 2011;6(6):e20661. 